PROCEEDINGS OF THE

AMERICAN MATHEMATICAL SOCIETY

Volume 130, Number 11, Pages 3209-3217

S 0002-9939(02)06466-3

Article electronically published on April 17, 2002

\title{
A NEW CONSTRUCTION OF THE KAC JORDAN SUPERALGEBRA
}

\author{
GEORGIA BENKART AND ALBERTO ELDUQUE
}

(Communicated by Lance W. Small)

To Irving Kaplansky

\begin{abstract}
We present an elementary construction of the 10-dimensional simple Jordan superalgebra $\mathrm{K}_{10}$ of Kac using the 3-dimensional tiny Kaplansky superalgebra. This new realization of $\mathrm{K}_{10}$ enables us to derive many of its properties.
\end{abstract}

\section{INTRODUCTION}

The 10-dimensional Jordan superalgebra $\mathrm{K}_{10}$ of $\mathrm{Kac}[\mathrm{K}$ is the only exceptional finite-dimensional simple Jordan superalgebra over an algebraically closed field of characteristic 0 . We give a new, elementary construction of it (over an arbitrary field of characteristic not 2) using the tiny Kaplansky superalgebra $K$. In our construction, $\mathrm{K}_{10}$ appears as a direct sum $F 1 \oplus(K \otimes K)$ with unit element 1 and with a suitable multiplication (see (2.1)). In characteristic $3, \mathrm{~K}_{10}$ is not simple but possesses a simple ideal $\mathrm{K}_{9}$ of dimension 9 , which is just the tensor product $K \otimes K$ (of superalgebras).

Our realization of $\mathrm{K}_{10}$ makes it easy to deduce certain of its known properties: for example, that its Lie superalgebra of superderivations is isomorphic to a direct sum of two copies of the orthosymplectic Lie superalgebra $\mathfrak{o s p}(1,2)$, or that its Lie multiplication superalgebra is $\mathfrak{o s p}(2,4)$ if -1 is a square in the field $F$.

The Kac superalgebra was originally constructed in $[\mathrm{K}$ by Lie theoretical methods from a 3-grading of the simple Lie superalgebra $F(4)$. No direct proof of the fact that it is a Jordan superalgebra is known. Indeed, in their work on Jordan superalgebras with semisimple even part, Racine and Zelmanov RZ remark "That $\mathrm{K}_{10}$, and hence $\mathrm{K}_{9}$, is a Jordan superalgebra can be obtained by Lie methods as in [K] but a direct proof would be desirable." Here we provide such a proof.

Received by the editors June 25, 2001.

2000 Mathematics Subject Classification. Primary 17A70, 17B60.

Key words and phrases. Kac superalgebra, Kaplansky superalgebra.

The first author gratefully acknowledges support from National Science Foundation Grant \#DMS-9970119.

The second author has been supported by the Spanish DGES (Pb 97-1291-C03-03) and by a grant from the Spanish Dirección General de Enseñanza Superior e Investigación Científica (Programa de Estancias de Investigadores Españoles en Centros de Investigación Extranjeros), while visiting the University of Wisconsin at Madison. 


\section{The KAPlansky SUPERALGEBRA}

Suppose $F$ is a field of characteristic not 2. The tiny Kaplansky superalgebra $\left(\mathrm{Kp}[\mathrm{M}]\right.$ ) is the 3 -dimensional Jordan superalgebra $K=K_{\overline{0}} \oplus K_{\overline{1}}$, with $K_{\overline{0}}=F e$ and $K_{\overline{1}}=F x \oplus F y$, and with multiplication given by

$$
\begin{gathered}
e^{2}=e, \quad \text { ex }=\frac{1}{2} x=x e, \quad \text { ey }=\frac{1}{2} y=y e, \\
x y=e=-y x, \quad x^{2}=0=y^{2} .
\end{gathered}
$$

It can be seen using (1.1) that the even supersymmetric bilinear form ( | ) defined on $K$ by

$$
(e \mid e)=\frac{1}{2}, \quad(x \mid y)=1, \quad \text { and } \quad\left(K_{\overline{0}} \mid K_{\overline{1}}\right)=0
$$

is associative $((a b \mid c)=(a \mid b c)$ for all $a, b, c \in K)$. Thus,

$$
(a b \mid c)=(-1)^{\bar{a}(\bar{b}+\bar{c})}(b c \mid a)=(-1)^{\bar{c}(\bar{a}+\bar{b})}(c a \mid b)
$$

for all $a, b, c \in K_{\overline{0}} \cup K_{\overline{1}}$. (Our convention is that $\bar{a}=i$ whenever $a \in K_{i}$.) Hence, since the supertrace, $\operatorname{str}\left(\mathrm{E}_{e}\right)=\operatorname{tr}_{K_{\overline{0}}}\left(\mathrm{E}_{e}\right)-\operatorname{tr}_{K_{\overline{1}}}\left(\mathrm{E}_{e}\right)$, of the left multiplication operator $\mathrm{E}_{e}$ of $e$ on $K$ is 0 , it follows that

$$
\begin{aligned}
\mathrm{E}_{K_{i}}=\{\phi & \in \operatorname{End}_{F}(K)_{i} \mid(\phi(u) \mid v) \\
& \left.=(-1)^{i \bar{u}}(u \mid \phi(v)) \quad \forall u, v \in K, \text { and } \operatorname{str}(\phi)=0\right\}
\end{aligned}
$$

for $i=\overline{0}, \overline{1}$. Moreover, $\left[\mathrm{E}_{K}, \mathrm{E}_{K}\right]=\operatorname{Der} K=\mathfrak{o s p}(K) \cong \mathfrak{o s p}(1,2)$.

Proposition 1.3. For all $u, v, w \in K_{\overline{0}} \cup K_{\overline{1}}$,

$$
(u v) w=(u \mid v) w+\frac{1}{2}\left(u(v \mid w)+(-1)^{\bar{u} \bar{v}} v(u \mid w)\right)
$$

Proof. Observe first that the expression in (1.4) is supersymmetric in $u$ and $v$. If $u=v=e$, then (1.4) reduces to $e w=\frac{1}{2} w+(e \mid w) e$, which is valid for all homogeneous elements $w$. If $u=e$ and $v \in K_{\overline{1}}$, then (1.4) becomes

$$
\frac{1}{2} v w=\frac{1}{2}(e(v \mid w)+v(e \mid w))
$$

which is true. Finally, if $u, v \in K_{\overline{1}}$, then $u v=(u \mid v) e$, and in this case, (1.4) says

$$
(u \mid v) e w=(u \mid v) w+\frac{1}{2}\left(u(v \mid w)+(-1)^{\bar{u} \bar{v}} v(u \mid w)\right) .
$$

For $w=e$ this reduces to $(u \mid v) e=(u \mid v) e$. If instead $w \in K_{\overline{1}}$, then (1.4) holds because $(u \mid v) w+(v \mid w) u+(w \mid u) v=0$, as the expression on the left is a skew-symmetric trilinear map on a 2 -dimensional vector space (namely $K_{\overline{1}}$ ).

Corollary 1.5. For all $u, v \in K_{\overline{0}} \cup K_{\overline{1}}$,

$$
\begin{gathered}
{\left[E_{u}, E_{v}\right]=\frac{1}{2}\left(u(v \mid-)-(-1)^{\bar{u} \bar{v}} v(u \mid-)\right) \quad \text { and }} \\
E_{u} \circ E_{v}=(u \mid v) \mathrm{I}+\frac{3}{2}\left(u(v \mid-)+(-1)^{\bar{u} \bar{v}} v(u \mid-)\right),
\end{gathered}
$$

where $\phi \circ \psi=\phi \psi+(-1)^{\bar{\phi} \bar{\psi}} \psi \phi$ for all homogeneous $\phi, \psi \in \operatorname{End}_{F}(K)$. 
Proof. From (1.4) we have

$$
\begin{aligned}
u(v w)-(-1)^{\bar{u}} \bar{v} v(u w)= & u(v \mid w)+\frac{1}{2}\left((u \mid v) w+(-1)^{\bar{u} \bar{v}} v(u \mid w)\right) \\
& \quad-(-1)^{\bar{u} \bar{v}}\left(v(u \mid w)+\frac{1}{2}\left((v \mid u) w+(-1)^{\bar{u} \bar{w}}(v \mid w) u\right)\right) \\
= & \frac{1}{2}\left(u(v \mid w)-(-1)^{\bar{u} \bar{v}} v(u \mid w)\right),
\end{aligned}
$$

which demonstrates (1.6). The verification of (1.7) is similar.

\section{The Jordan superalgebra $J$}

In this section we consider the superalgebra

$$
J=F 1 \oplus(K \otimes K)
$$

with unit element 1 and with product defined by

$$
(a \otimes b)(c \otimes d)=(-1)^{\bar{b} \bar{c}}\left(a c \otimes b d-\frac{3}{4}(a \mid c)(b \mid d) 1\right)
$$

for homogeneous elements $a, b, c, d \in K$. (All tensor products are over $F$ unless specified otherwise.) By its definition $J$ is a (super-)commutative superalgebra. Our aim is to show that $J$ is a Jordan superalgebra. First we establish some properties of the multiplication.

Proposition 2.2. For homogeneous elements $a, b, c, d \in K$,

$$
\begin{aligned}
& {\left[E_{a \otimes b}, E_{c \otimes d}\right](1)=0} \\
& {\left.\left[E_{a \otimes b}, E_{c \otimes d}\right]\right|_{K \otimes K}=(-1)^{\bar{b} \bar{c}} \frac{1}{2}\left(\left[E_{a}, E_{c}\right] \otimes(b \mid d) \mathrm{I}+(a \mid c) \mathrm{I} \otimes\left[E_{b}, E_{d}\right]\right) .}
\end{aligned}
$$

Proof. First let us remark that

$$
(\phi \otimes \psi)(u \otimes v)=(-1)^{\bar{\psi} \bar{u}} \phi(u) \otimes \psi(v)
$$

for homogeneous $\phi, \psi \in \operatorname{End}_{F}(K), u, v \in K$. The first equation in (2.3) is clear by supercommutativity. As for the second, note that

$$
\begin{aligned}
{\left[\mathrm{E}_{a \otimes b}, \mathrm{E}_{c \otimes d}\right](u \otimes v) } & \\
= & (-1)^{\bar{d} \bar{u}}(a \otimes b)\left(c u \otimes d v-\frac{3}{4}(c \mid u)(d \mid v) 1\right) \\
& -(-1)^{(\bar{a}+\bar{b})(\bar{c}+\bar{d})}(-1)^{\bar{b} \bar{u}}(c \otimes d)\left(a u \otimes b v-\frac{3}{4}(a \mid u)(b \mid v) 1\right) \\
= & (-1)^{\bar{d} \bar{u}}(-1)^{\bar{b}(\bar{c}+\bar{u})}\left(a(c u) \otimes b(d v)-\frac{3}{4}(a \mid c u)(b \mid d v) 1-\frac{3}{4} a(c \mid u) \otimes b(d \mid v)\right. \\
& \left.-(-1)^{\bar{a} \bar{c}+\bar{b} \bar{d}}\left(c(a u) \otimes d(b v)-\frac{3}{4}(c \mid a u)(d \mid b v) 1-\frac{3}{4} c(a \mid u) \otimes d(b \mid v)\right)\right) .
\end{aligned}
$$


By associativity $(a \mid c u)=(-1)^{\bar{a} \bar{c}}(c \mid a u)$ and $(b \mid d v)=(-1)^{\bar{b}} \bar{d}(d \mid b v)$, so that the F1-component of (2.4) is 0 . Moreover,

$$
\begin{aligned}
a(c u) \otimes b(d v)- & (-1)^{\bar{a} \bar{c}+\bar{b} \bar{d}} c(a u) \otimes d(b v) \\
= & \frac{1}{2}\left(\left[\mathrm{E}_{a}, \mathrm{E}_{c}\right](u) \otimes\left(\mathrm{E}_{b} \circ \mathrm{E}_{d}\right)(v)+\left(\mathrm{E}_{a} \circ \mathrm{E}_{c}\right)(u) \otimes\left[\mathrm{E}_{b}, \mathrm{E}_{d}\right](v)\right),
\end{aligned}
$$

and by (1.6),

$$
\begin{aligned}
& a(c \mid u) \otimes b(d \mid v)-(-1)^{\bar{a} \bar{c}+\bar{b} \bar{d}} c(a \mid u) \otimes d(b \mid v) \frac{1}{2}\left(a(c \mid u)-(-1)^{\bar{a} \bar{c}} c(a \mid u)\right) \otimes\left(b(d \mid v)+(-1)^{\bar{b} \bar{d}} d(b \mid v)\right) \\
& \quad+\left(a(c \mid u)+(-1)^{\bar{a} \bar{c}} c(a \mid u)\right) \otimes\left(b(d \mid v)-(-1)^{\bar{b} \bar{d}} d(b \mid v)\right) \\
&=\left[\mathrm{E}_{a}, \mathrm{E}_{c}\right](u) \otimes\left(b(d \mid v)+(-1)^{\bar{b}} \bar{d} d(b \mid v)\right) \\
& \quad+\left(a(c \mid u)+(-1)^{\bar{a} \bar{c}} c(a \mid v)\right) \otimes\left[\mathrm{E}_{b}, \mathrm{E}_{d}\right](v) .
\end{aligned}
$$

Therefore, it follows from (1.7) that

$$
\begin{aligned}
{\left.\left[\mathrm{E}_{a \otimes b}, \mathrm{E}_{c \otimes d}\right]\right|_{K \otimes K} } & \\
= & (-1)^{\bar{b} \bar{c}}\left(\left[\mathrm{E}_{a}, \mathrm{E}_{c}\right] \otimes\left(\frac{1}{2} \mathrm{E}_{b} \circ \mathrm{E}_{d}-\frac{3}{4}\left(b(d \mid-)+(-1)^{\bar{b} \bar{d}} d(b \mid-)\right)\right)\right. \\
& \left.+\left(\frac{1}{2} \mathrm{E}_{a} \circ \mathrm{E}_{c}-\frac{3}{4}\left(a(c \mid-)+(-1)^{\bar{a} \bar{c}} c(a \mid-)\right)\right) \otimes\left[\mathrm{E}_{b}, \mathrm{E}_{d}\right]\right) \\
= & (-1)^{\bar{b} \bar{c}} \frac{1}{2}\left(\left[\mathrm{E}_{a}, \mathrm{E}_{c}\right] \otimes(b \mid d) \mathrm{I}+(a \mid c) \mathrm{I} \otimes\left[\mathrm{E}_{b}, \mathrm{E}_{d}\right]\right),
\end{aligned}
$$

as desired.

Corollary 2.5. For $a, b, c, d \in K_{\overline{0}} \cup K_{\overline{1}},\left[E_{a \otimes b}, E_{c \otimes d}\right]$ is a superderivation of $J$.

Proof. Because $K$ is a Jordan superalgebra, for $a, b \in K_{\overline{0}} \cup K_{\overline{1}}$, the mapping $\left[\mathrm{E}_{a}, \mathrm{七}_{b}\right]$ is a superderivation of $K$. By the associativity of $(\mid)$, it is super-skew-symmetric relative to $(\mid)$. Consequently, from (2.3) we can conclude that $\left[\mathrm{E}_{a \otimes b}, \mathrm{E}_{c \otimes d}\right]$ is a superderivation of $J$.

Theorem 2.6. $J=F 1 \oplus(K \otimes K)$ is a Jordan superalgebra if char $F \neq 2$.

Proof. A Jordan algebra over a field $F$ of characteristic not 2 or 3 is characterized by commutativity and the identity $\sum_{\text {cyclic }}\left[\mathrm{E}_{u v}, \mathrm{E}_{w}\right]=0$. Hence a Jordan superalgebra over $F$ is characterized by supercommutativity and

$$
\sum_{\text {supercyclic }}\left[\mathrm{E}_{u v}, \mathrm{E}_{w}\right]=\left[\mathrm{E}_{u v}, \mathrm{E}_{w}\right]+(-1)^{\bar{u}(\bar{v}+\bar{w})}\left[\mathrm{E}_{v w}, \mathrm{E}_{u}\right]+(-1)^{(\bar{u}+\bar{v}) \bar{w}}\left[\mathrm{E}_{w u}, \mathrm{E}_{v}\right]=0 .
$$


In the supercommutative algebra $J$, we have from (1.2) and (2.3) that for all homogeneous $a, b, c, d, u, v \in K$,

$$
\begin{aligned}
\sum_{\text {supercyclic }} & {\left[\mathrm{E}_{(a \otimes b)(c \otimes d)}, \mathrm{E}_{u \otimes v}\right] } \\
& =\sum_{\text {supercyclic }}(-1)^{\bar{b} \bar{c}}\left[\mathrm{E}_{a c \otimes b d}, \mathrm{E}_{u \otimes v}\right] \\
& =\sum_{\text {supercyclic }}(-1)^{\bar{b}(\bar{c}+\bar{u})+\bar{d} \bar{v}} \frac{1}{2}\left(\left[\mathrm{E}_{a c}, \mathrm{E}_{u}\right] \otimes(b d \mid v) \mathrm{I}+(a c \mid u) \mathrm{I} \otimes\left[\mathrm{E}_{b d}, \mathrm{E}_{v}\right]\right) \\
& =(-1)^{\bar{b}(\bar{c}+\bar{u})+\bar{d} \bar{u}} \frac{1}{2}\left\{\left(\sum_{\text {supercyclic }}\left[\mathrm{E}_{a c}, \mathrm{E}_{u}\right]\right) \otimes(b d \mid v) \mathrm{I}\right. \\
& \left.+(a c \mid u) \mathrm{I} \otimes\left(\sum_{\text {supercyclic }}\left[\mathrm{E}_{b d}, \mathrm{E}_{v}\right]\right)\right\} \\
& 0 .
\end{aligned}
$$

Thus, $J$ is a Jordan superalgebra whenever the field has characteristic different from 2 or 3 .

If $R$ is the ring of fractions $R=S^{-1} \mathbb{Z}$ relative to the multiplicative set of integers $S=\left\{2^{n} \mid n=0,1, \ldots\right\}$, we could have defined $K$ and $J$ over $R$ as above. (Denote the result by $J_{R}$.) Then $J_{\mathbb{Q}}=\mathbb{Q} \otimes_{R} J_{R}$ is a Jordan superalgebra over the rationals $\mathbb{Q}$, and so is $J_{R}$ over $R$. But if $F$ is a field having characteristic not 2 , then $J=F \otimes_{R} J_{R}$ is a Jordan superalgebra over $F$.

From the computations and results above, it is easy to draw the following conclusions about the Jordan superalgebra $J$. Here we use the associator $(u, v, w)=$ $(u v) w-u(v w)$ in $J$ in the statements.

Proposition 2.7. For the Jordan superalgebra $J=F 1 \oplus(K \otimes K)$ :

(a) $(J, J, J)=K \otimes K$.

(b) $J=F 1 \oplus(J, J, J)$.

(c) If char $F=3$, then $K \otimes K=(J, J, J)$ is an ideal of $J$.

(d) $e_{1}:=-\frac{1}{2} 1+2 e \otimes e$ and $e_{2}:=\frac{3}{2} 1-2 e \otimes e$ are orthogonal idempotents in $J$. Relative to $e_{1}$ the Peirce spaces $J^{(\lambda)}:=\left\{u \in J \mid e_{1} u=\lambda u\right\}$ are given by

$$
J^{(1)}=F e_{1}, \quad J^{(0)}=F e_{2} \oplus\left(K_{\overline{1}} \otimes K_{\overline{1}}\right), \quad J^{\left(\frac{1}{2}\right)}=\left(e \otimes K_{\overline{1}}\right) \oplus\left(K_{\overline{1}} \otimes e\right) .
$$

Thus, $J_{\overline{0}}=J^{(1)} \oplus J^{(0)}$ and $J_{\overline{1}}=J^{\left(\frac{1}{2}\right)}$. The space $J^{(0)}$ is the Jordan algebra of a nondegenerate symmetric bilinear form, and $e_{2}$ is its unit element.

Theorem 2.8. (a) If char $F \neq 2,3, \quad J=F 1 \oplus(K \otimes K)$ is a simple Jordan superalgebra.

(b) If char $F=3,(J, J, J)=K \otimes K$ is a simple Jordan superalgebra.

(c) Der $J=$ Inder $J$ and it is isomorphic to $\mathfrak{o s p}(1,2) \oplus \mathfrak{o s p}(1,2)$.

Proof. We begin with (c). Any superderivation of $J$ annihilates $F 1$ and leaves invariant $(J, J, J)=K \otimes K$. Therefore, using the natural identifications and the fact that Der $K=$ Inder $K \cong \mathfrak{o s p}(1,2)$, we have by $(2.3)$ that

$$
\text { Der } K \oplus \operatorname{Der} K \cong(\operatorname{Der} K \otimes \mathrm{I}) \oplus(\mathrm{I} \otimes \operatorname{Der} K)=\text { Inder } J \text {. }
$$


Now one may check that Inder $J=\operatorname{Der} J$ as follows: First for any $D \in(\operatorname{Der} J)_{\overline{0}}$, $\left.D\right|_{J_{\overline{0}}} \in \operatorname{Der}\left(J_{\overline{0}}\right)$. Since $J_{\overline{0}}$ is a direct sum of a 1-dimensional ideal $J^{(1)}=F e_{1}$ and the Jordan algebra $J^{(0)}=F e_{2} \oplus\left(K_{\overline{1}} \otimes K_{\overline{1}}\right)$ of a bilinear form, $\operatorname{Der}\left(J_{\overline{0}}\right)=$ Inder $J_{\overline{0}}$. Thus, there is a $\tilde{D} \in(\operatorname{Inder} J)_{\overline{0}}$ with $(D-\tilde{D})\left(J_{\overline{0}}\right)=0$. It follows easily that $D=\tilde{D}$. Now suppose $E \in(\operatorname{Der} J)_{\overline{1}}$. Then there is a $\tilde{E} \in(\text { Inder } J)_{\overline{1}}$ with $E\left(e_{1}\right)=\tilde{E}\left(e_{1}\right)$ because (Inder $J)_{\overline{1}} e_{1}=J_{\overline{1}}$. For any $z \in J_{\overline{1}}, e_{1} z=\frac{1}{2} z$, so $(E-\tilde{E})(z)=2(E-\tilde{E})\left(e_{1} z\right)=$ $2 e_{1}(E-\tilde{E})(z)$. Thus, $(E-\tilde{E})(z) \in J_{\overline{0}} \cap J^{\left(\frac{1}{2}\right)}=0$, so that $(E-\tilde{E})\left(J_{\overline{1}}\right)=0$. As a consequence, $E=\tilde{E}$ and $(\operatorname{Der} J)_{\overline{1}}=(\operatorname{Inder} J)_{\overline{1}}$.

Since Der $K$ acts irreducibly on $K$, so does Der $J=\operatorname{Inder} J$ on $K \otimes K=(J, J, J)$, which is an ideal of $J$ if and only if the characteristic of $F$ is 3. From this (a) and (b) follow.

The Lie multiplication superalgebra of $J$. To compute the Lie multiplication superalgebra of $J$ we need some preliminaries. Let $H$ be a unital Jordan superalgebra with a normalized trace, that is, a linear map $\mathfrak{t}: H \rightarrow F$ such that $\mathfrak{t}(1)=1$ and $\mathfrak{t}((H, H, H))=0$. Assume that $(H, H, H) \neq 0$. Let $H_{0}=$ ker $\mathfrak{t}$. Then for any $x, y \in H_{0}, x y=\mathfrak{t}(x y) 1+x * y$, where $x * y=x y-\mathfrak{t}(x y) 1$. The Lie multiplication superalgebra is the Lie superalgebra spanned by $\mathrm{L}_{H}$, which is a direct sum of the central ideal $F \mathrm{I}$ and the ideal $\mathfrak{L}_{0}(H)=\mathrm{E}_{H_{0}} \oplus\left[\mathrm{E}_{H_{0}}, \mathrm{七}_{H_{0}}\right]$. For simplicity, let us adopt the notation $S=$ Inder $H$ and $T=H_{0}$. Then $\mathfrak{L}_{0}(H)$ is isomorphic to the Lie superalgebra $\mathfrak{L}=S \oplus T$ with multiplication determined by

(1) the natural Lie multiplication on $S$,

(2) the natural action of $S$ on $T$, that is, $[s, t]=s(t)$ for any $s \in S$ and $t \in T$, and

(3) $\left[t_{1}, t_{2}\right]=\left[\mathrm{Ł}_{t_{1}}, \mathrm{Ł}_{t_{2}}\right] \in S$, for any $t_{1}, t_{2} \in T$.

Also, given a nonzero scalar $\nu \in F$, let $\mathfrak{L}_{\nu}$ be the Lie superalgebra defined over $\mathfrak{L}$, but with the new bracket given by

(i) $[x, y]^{\nu}=[x, y]$, for $x, y$ both in $S$ or one in $S$ and the other in $T$,

(ii) $[x, y]^{\nu}=\nu[x, y]$, for $x, y \in T$.

Lemma 2.10. Under the hypotheses above, if $\operatorname{Hom}_{S}(T \otimes T, T)$ is spanned by $u \otimes$ $v \mapsto u * v$ and if any automorphism of the superalgebra $(T, *)$ is an isometry of the supersymmetric bilinear form $(u, v) \mapsto \mathfrak{t}(u v)$, then for any $0 \neq \mu, \nu \in F, \mathfrak{L}_{\mu}$ is isomorphic to $\mathfrak{L}_{\nu}$ if and only if $\mu^{-1} \nu \in F^{2}$.

Proof. It is enough to apply the ideas of ( $\mathrm{BDE}$, proof of Prop. 4.2). We include the argument for completeness.

First, since any isomorphism $\mathfrak{L}_{\mu} \rightarrow \mathfrak{L}_{\nu}$ is also an isomorphism $\mathfrak{L} \rightarrow \mathfrak{L}_{\mu^{-1}}$, it is enough to assume $\mu=1$. Suppose $\Phi: \mathfrak{L} \rightarrow \mathfrak{L}_{\nu}$ is an isomorphism. Then the map $T \otimes T \rightarrow T$ defined by $u \otimes v \mapsto \Phi^{-1}(\Phi(u) * \Phi(v))$ is $S$-invariant. Consequently, there exists a nonzero scalar $\alpha \in F$ such that $\alpha \Phi(u) * \Phi(v)=\Phi(u * v)$ for any $u, v \in T$. Then $\psi: T \rightarrow T$ given by $\psi(u)=\alpha \Phi(u)$ is an automorphism of $(T, *)$ which, by hypothesis, extends by means of $\psi(1)=1$ to an automorphism of the Jordan superalgebra $H$. Now the map $\Psi: \mathfrak{L} \rightarrow \mathfrak{L}$, such that $\Psi(s)=\psi s \psi^{-1}$ (composition of maps) for $s \in S$, and $\Psi(t)=\psi(t)$ for $t \in T$ is an automorphism of $\mathfrak{L}$. 
Furthermore, $\tilde{\Phi}=\Phi \Psi^{-1}: \mathfrak{L} \rightarrow \mathfrak{L}_{\nu}$ is an isomorphism such that $\tilde{\Phi}(t)=$ $\Phi\left(\psi^{-1}(t)\right)=\alpha^{-1} t$ for $t \in T$. Hence, for any $t_{1}, t_{2}, t_{3} \in T$,

$$
\begin{aligned}
\tilde{\Phi}\left(\left[t_{1}, t_{2}\right]\right) & =\left[\tilde{\Phi}\left(t_{1}\right), \tilde{\Phi}\left(t_{2}\right)\right]^{\nu}=\alpha^{-2} \nu\left[t_{1}, t_{2}\right], \\
\alpha^{-1}\left[\left[t_{1}, t_{2}\right], t_{3}\right] & =\tilde{\Phi}\left(\left[\left[t_{1}, t_{2}\right], t_{3}\right]\right) \\
& =\left[\left[\tilde{\Phi}\left(t_{1}\right), \tilde{\Phi}\left(t_{2}\right)\right]^{\nu}, \tilde{\Phi}\left(t_{3}\right)\right]^{\nu} \\
& =\alpha^{-3} \nu\left[\left[t_{1}, t_{2}\right], t_{3}\right],
\end{aligned}
$$

so that $\nu=\alpha^{-2}$ as required. (Note $[[T, T], T] \neq 0$ because $(H, H, H) \neq 0$.)

The converse is clear, since the map $\Phi$ given by $\Phi(s)=s$ for $s \in S$ and $\Phi(t)=\alpha t$ for $t \in T$ provides an isomorphism $\mathfrak{L} \rightarrow \mathfrak{L}_{\alpha^{2}}$.

In order to apply the last result to our 10-dimensional Jordan superalgebra $J$, notice first that $J$ is endowed with a normalized trace $\mathfrak{t}$ such that $\mathfrak{t}(1)=1$ and $\mathfrak{t}((J, J, J))=0$, as $(J, J, J)=K \otimes K$ does not intersect $F 1$. Moreover, the associated bilinear form is given by $\mathfrak{t}((a \otimes b)(c \otimes d))=-(-1)^{\bar{b} \bar{c}} \frac{3}{4}(a \mid c)(b \mid d)$ for any homogeneous $a, b, c, d \in K$, because of (2.1). Then all we need is the following

Lemma 2.11. Any automorphism of $(K \otimes K, *)$ is an isometry of the bilinear trace form.

Proof. Write $T=J_{0}=K \otimes K$ and set $\langle a \otimes b \mid c \otimes d\rangle=(-1)^{\bar{b} \bar{c}}(a \mid c)(b \mid d)$ for any homogeneous elements $a, b, c, d \in K$. Then $T_{\overline{0}}=F(e \otimes e) \oplus\left(K_{\overline{1}} \otimes K_{\overline{1}}\right)$. Suppose $\tilde{e}=e \otimes e$. Then the multiplication in $T_{\overline{0}}$ is given by

$$
\tilde{e}^{* 2}=\tilde{e}, \quad \tilde{e} * v=\frac{1}{4} v, \quad v * w=\langle v \mid w\rangle \tilde{e}
$$

for all $v, w \in K_{\overline{1}} \otimes K_{\overline{1}}$.

Let $\varphi$ be an automorphism of $(T, *)$ and let us first verify that $\varphi(\tilde{e})=\tilde{e}$. This is clear if the characteristic is 3 , because $\tilde{e}$ is then the unit element of $T_{\overline{0}}$. Otherwise, $\tilde{e}$ is the only idempotent of $T_{\overline{0}}$ such that the corresponding left multiplication operator has eigenvalue $\frac{1}{4}$ with multiplicity 4 . This is because the eigenvalues of $\mathrm{E}_{\alpha \tilde{e}+v}$ (for $v \in K_{\overline{1}} \otimes K_{\overline{1}}, \alpha \in F$ ) include $\frac{\alpha}{4}$ with multiplicity at least 3 , as any $w \in K_{\overline{1}} \otimes K_{\overline{1}}$ which is orthogonal to $v$ relative to $\langle\mid\rangle$ is a corresponding eigenvector. Moreover, $\tilde{e}+v$ is an idempotent only if $v=0$.

Now, $\varphi\left(K_{\overline{1}} \otimes K_{\overline{1}}\right) \subseteq K_{\overline{1}} \otimes K_{\overline{1}}$ because $K_{\overline{1}} \otimes K_{\overline{1}}=\left\{v \in T_{\overline{0}} \mid \tilde{e} \notin F v\right.$ and $\left.v * v \in F \tilde{e}\right\}$. Because of $(2.12),\left.\varphi\right|_{T_{\overline{0}}}$ is an isometry of the restriction of $\langle\mid\rangle$ to $T_{\overline{0}}$.

Finally, $\left(K_{\overline{1}} \otimes e\right) \cup\left(e \otimes K_{\overline{1}}\right)=\left\{z \in T_{\overline{1}} \mid z * z \in F \tilde{e}\right\}$, and $K_{\overline{1}} \otimes e$ and $e \otimes K_{\overline{1}}$ are the only 2-dimensional subspaces contained in this union. Therefore, either $\varphi$ preserves $K_{\overline{1}} \otimes e$ and $e \otimes K_{\overline{1}}$ or it interchanges them. Since $(u \otimes e) *(v \otimes e)=\frac{1}{2}\langle u \otimes e \mid v \otimes e\rangle \tilde{e}$ and $(e \otimes u) *(e \otimes v)=\frac{1}{2}\langle e \otimes u \mid e \otimes v\rangle \tilde{e}$ for any $u, v \in K_{\overline{1}}$, it follows that $\left.\varphi\right|_{T_{\overline{1}}}$ is an isometry too.

We are ready to determine the Lie multiplication superalgebra $\mathfrak{L}_{0}(J)$ for $J=$ $F 1 \oplus(K \otimes K)$. When the underlying field $F$ has characteristic 0 , the next result is stated in Sh] but without the assumption of -1 being a square in $F$.

Theorem 2.13. $\mathfrak{L}_{0}(J)_{-1} \cong \mathfrak{o s p}(K \oplus K)(\cong \mathfrak{o s p}(2,4))$. In particular, $\mathfrak{L}_{0}(J)$ is a form of $\mathfrak{o s p}(2,4)$ and $\mathfrak{L}_{0}(J) \cong \mathfrak{o s p}(2,4)$ if and only if -1 is a square in $F$. 
Proof. On any vector superspace $V$ equipped with a supersymmetric bilinear form ( $\mid)$, the transformation $\gamma_{u, v}=u(v \mid-)-(-1)^{\bar{u} \bar{v}} v(u \mid-)$ belongs to $\mathfrak{o s p}(V)$ for all homogeneous $u, v \in V$. Because of (2.3) and (1.7), Der $J$ is embedded in $\mathfrak{o s p}(K \oplus K)$ (with the natural supersymmetric bilinear form on $K \oplus K$ that makes this an orthogonal sum) by means of

$$
\Phi\left(\left[\mathrm{E}_{a \otimes b}, \mathrm{E}_{c \otimes d}\right]\right)=(-1)^{\bar{b} \bar{c}} \frac{1}{4}\left(\gamma_{(a, 0),(c, 0)}(b \mid d)+(a \mid c) \gamma_{(0, b),(0, d)}\right) \in \mathfrak{o s p}(K \oplus K) .
$$

This can be extended to a linear bijection $\Phi: \mathfrak{L}_{0}(J)_{-1} \rightarrow \mathfrak{o s p}(K \oplus K)$ by defining

$$
\Phi\left(\mathrm{E}_{a \otimes b}\right)=\frac{1}{2} \gamma_{(a, 0),(0, b)}
$$

for $a, b \in K$. Since

$$
\begin{aligned}
{\left[\gamma_{(a, 0),(0, b)},\right.} & \left.\gamma_{(c, 0),(0, d)}\right] \\
& =\gamma_{\gamma_{(a, 0),(0, b)}(c, 0),(0, d)}+(-1)^{(\bar{a}+\bar{b}) \bar{c}} \gamma_{(c, 0), \gamma_{(a, 0),(0, b)}(0, d)} \\
& =-(-1)^{\bar{a} \bar{b}}(-1)^{(\bar{a}+\bar{c}) \bar{b}}(a \mid c) \gamma_{(0, b),(0, d)}+(-1)^{(\bar{a}+\bar{b}) \bar{c}} \gamma_{(c, 0),(a, 0)}(b \mid d) \\
& =-(-1)^{\bar{b} \bar{c}}\left((a \mid c) \gamma_{(0, b),(0, d)}+\gamma_{(a, 0),(c, 0)}(b \mid d)\right)
\end{aligned}
$$

it follows that $\Phi$ is an isomorphism of Lie superalgebras. The last part is a consequence of Lemmata 2.10 and 2.11 .

\section{The KAC SUPERALGEBRA}

We may identify the simple Jordan superalgebra $J$ with the 10 -dimensional simple Jordan superalgebra $K_{10}$ discovered by Kac [K] (see also [HK]) when the characteristic is not 2 or 3 , and $(J, J, J)=K \otimes K$ with the 9-dimensional degenerate Kac superalgebra $K_{9}$ if $\operatorname{char} F=3$. This realization may be described explicitly as follows. In the notation of [MZ] or [RZ], we suppose $\left\{\check{e}, v_{1}, v_{2}, v_{3}, v_{4}, \check{f}, x_{1}, y_{1}, x_{2}, y_{2}\right\}$ is a basis of $\mathrm{K}_{10}$ with $\left(\mathrm{K}_{10}\right)_{\overline{0}}=\operatorname{span}_{F}\left\{\check{e}, v_{1}, v_{2}, v_{3}, v_{4}, \check{f}\right\}$ and $\left(\mathrm{K}_{10}\right)_{\overline{1}}=\operatorname{span}_{F}\left\{x_{1}, y_{1}\right.$, $\left.x_{2}, y_{2}\right\}$ and with multiplication given by

$$
\begin{gathered}
\check{e}^{2}=\check{e}, \quad \check{e} v_{i}=v_{i}, \quad v_{1} v_{2}=2 \check{e}=v_{3} v_{4}, \quad \check{f}^{2}=\check{f}, \\
\check{e} x_{j}=\frac{1}{2} x_{j}=\check{f} x_{j}, \quad \check{e} y_{j}=\frac{1}{2} y_{j}=\check{f} y_{j}, \\
v_{1} y_{1}=x_{2}, \quad v_{1} y_{2}=-x_{1}, \quad v_{2} x_{1}=-y_{2}, \quad v_{2} x_{2}=y_{1}, \\
v_{3} x_{2}=x_{1}, \quad v_{3} y_{1}=y_{3}, \quad v_{4} x_{1}=x_{2}, \quad v_{4} y_{2}=y_{1}, \\
x_{j} y_{j}=\check{e}-3 \check{f}, \quad x_{1} x_{3}=v_{1}, \quad x_{1} y_{2}=v_{3}, \quad x_{2} y_{1}=v_{4}, \quad y_{1} y_{2}=v_{2}
\end{gathered}
$$

for $i=1,2,3,4$ and $j=1,2$. All other products are either 0 or obtained from those in (3.1) by supercommutativity. Then a straightforward computation using (3.1) shows that the assignment

$$
\begin{array}{cl}
\check{e} \leftrightarrow \frac{3}{2} 1-2 e \otimes e=e_{2} & \\
\check{f} \leftrightarrow-\frac{1}{2} 1+2 e \otimes e=e_{1} & x_{1} \leftrightarrow 4 x \otimes e \\
v_{1} \leftrightarrow-4 x \otimes x & x_{2} \leftrightarrow-4 e \otimes x \\
v_{2} \leftrightarrow-y \otimes y & y_{1} \leftrightarrow-2 y \otimes e \\
v_{3} \leftrightarrow 2 x \otimes y & y_{2} \leftrightarrow 2 e \otimes y \\
v_{4} \leftrightarrow-2 y \otimes x &
\end{array}
$$


gives an isomorphism between $\mathrm{K}_{10}$ and $J$. Additionally, if $\operatorname{char} F=3$, then $\check{e} \leftrightarrow$ $-2 e \otimes e$, so that the isomorphism restricts to an isomorphism $\mathrm{K}_{9} \leftrightarrow K \otimes K$.

Concluding remarks. As the reader may have guessed, the actual process for deriving our results was the reverse path of the presentation above. First it was verified that $\mathfrak{g}:=\operatorname{Der} \mathrm{K}_{10}=\mathfrak{o} \mathfrak{s p}(1,2) \oplus \mathfrak{o s p}(1,2)$, (compare Shtern Sh), and that as module for the Lie superalgebra $\mathfrak{g}, \quad \mathrm{K}_{10}=F 1 \oplus(V \otimes V)$, where $V$ is the natural 3-dimensional module for $\mathfrak{o s p}(1,2)$. The first copy of $\mathfrak{o s p}(1,2)$ operates on the first tensor slot of $V \otimes V$, and the second copy on the second slot. The spaces $\operatorname{Hom}_{\mathfrak{o s p}(1,2)}(V \otimes V, F)$ and $\operatorname{Hom}_{\mathfrak{o s p}(1,2)}(V \otimes V, \mathfrak{o s p}(1,2))$ are 1-dimensional. By identifying $V$ with the tiny Kaplansky superalgebra $K$, we may describe the generators of these spaces nicely by the maps $u \otimes v \mapsto(u \mid v)$ and $u \otimes v \mapsto\left[\mathrm{E}_{u}, \mathrm{E}_{v}\right] \in \mathfrak{o s p}(1,2)$ for $u, v \in K$. From this, the multiplication in the Kac superalgebra $\mathrm{K}_{10}$ is almost uniquely determined in terms of $K$.

In [S1], Shestakov proposed quite a different realization of the superalgebra $\mathrm{K}_{10}$ in characteristic $\neq 2,3$, but the same description of $\mathrm{K}_{9}$; however, he has not published this work [S2].

\section{REFERENCES}

[BDE] P. Benito, C. Draper and A. Elduque, On some algebras related to simple Lie triple systems, J. Algebra 219 (1999), 234-254. MR 2000i:17005

[HK] L. Hogben and V.G. Kac, Erratum: Classification of simple $\mathbb{Z}$-graded Lie superalgebras and simple Jordan superalgebras, Comm. Algebra 5 (1977), 1375-1400, Comm. Algebra 11 (1983), 1155-1156. MR 58:16806; MR 85a:17011

[K] V.G. Kac, Classification of $\mathbb{Z}$-graded Lie superalgebras and simple Jordan superalgebras, Comm. Algebra 5 (1977), 1375-1400. MR 58:16806

[Kp] I. Kaplansky, Graded Jordan Algebras I, preprint.

[M] K. McCrimmon, Kaplansky superalgebras, J. Algebra 164 (1994), 656-694. MR 95e: 17002

[MZ] C. Martínez and E. Zelmanov, Simple finite dimensional Jordan superalgebras of prime characteristic, J. Algebra 236 (2001), 575-629. CMP 2001:08

[RZ] M. Racine and E. Zelmanov, Classification of simple Jordan superalgebras with semisimple even part, to appear.

[S1] I.P. Shestakov, Lecture, Jordan Algebren Conference, Oberwolfach, Germany, 1996.

[S2] I.P. Shestakov, private communication, June 2001.

[Sh] A.S. Shtern, Representations of an exceptional Jordan superalgebra, Funktsional Anal. i Prilozhen 21 (1987), 93-94 (Russian); English transl. in Functional Anal. Appl. 21 (1987), 253-254. MR 89b:17009

Department of Mathematics, University of Wisconsin, Madison, Wisconsin 53706

E-mail address: benkart@math.wisc.edu

Departamento de Matemáticas, Universidad de Zaragoza, 50009 Zaragoza, Spain

E-mail address: elduque@posta.unizar.es 